\title{
Der Placeboeffekt in der Schmerztherapie - für den klinischen Alltag nutzbar? Ja, unbedingt!
}

\section{The Placebo Effect in Pain Therapy - Suitable for Everyday Clinical Use? Yes, Definitely!}

Autoren

Julian Kleine-Borgmann (D, Ulrike Bingel

Institut

Klinik für Neurologie, Universitätsmedizin Essen, Essen

Schlüsselwörter

Placebo-Effekt, Schmerzmedizin, Placeboanalgesie

Key words

Placebo effect, Pain management, Placeboanalgesia

Online publiziert: 26.8 .2020

Bibliografie

Akt Rheumatol 2020; 45: 453-459

DOI 10.1055/a-1165-6689

ISSN 0341-051X

(c) 2020. Thieme. All rights reserved.

Georg Thieme Verlag KG, Rüdigerstraße 14,

70469 Stuttgart, Germany

Korrespondenzadresse

Dr. Julian Kleine-Borgmann

Klinik für Neurologie

Universitätsmedizin Essen

Hufelandstraße 55

45147 Essen

Tel.: 00492017232446 , Fax : 004902017236882

julian.kleine-borgmann@uk-essen.de

\section{ZUSAMMENFASSUNG}

Als Placeboeffekte bezeichnet man positive physiologische oder psychologische Veränderungen, die nach einer Einnahme von Medikamenten ohne spezifischen Wirkstoff oder Scheineingriffen auftreten und durch Erwartungs- und Konditionie- rungsprozesse induziert werden. Die mit Placeboeffekten einhergehenden neurobiologischen und peripher-physiologischen Vorgänge können auch bei der Vergabe von wirkstoffhaltigen Medikamenten eine wichtige Rolle spielen und sind somit nicht zwingend an die Gabe eines Scheinmedikamentes gebunden. Die Darreichung von „traditionellen“ Placebos ist im klinischen Alltag aus juristischen und ethischen Gründen höchst problematisch. Dieser Beitrag zeigt neben neuen Erkenntnissen zu offenen Placebo-Anwendungen (Open-Label Placebos) Wege auf, wie die zugrundeliegenden Mechanismen von Placeboantworten dennoch gezielt im schmerztherapeutischen Alltag genutzt werden können, um die Wirksamkeit, Verträglichkeit und Compliance bestehender pharmakologischer und anderer Therapien zum Wohle des Patienten zu optimieren.

\section{ABSTRACT}

Placebo effects are defined as positive physiological or psychological changes that occur after either taking pills without a specific active ingredient or after receiving sham interventions. The underlying mechanisms are based on expectation and conditioning effects. The neurobiological and peripheral physiological processes associated with placebo effects can also play an important role in pharmacological treatments and are therefore not necessarily linked to the use of a sham drug or intervention. In clinical settings, the use of "traditional" placebos is highly problematic for both legal and ethical reasons. In addition to recent scientific findings on open-label placebo applications, this article offers suggestions on how to harness analgesic placebo effects by utilising their underlying mechanisms to optimise the effectiveness, tolerability and compliance of existing pharmacological and non-pharmacological treatments for the patient's benefit.

\section{Placebos und Schmerztherapie: Ein Fallbeispiel}

Auf einer Normalstation befindet sich eine 52-jährige Patientin, die aufgrund einer Zunahme ihrer Fibromyalgie bedingten Schmerzen den diensthabenden Arzt der Notaufnahme in der Nacht um Hilfe bittet. Die bestehende Schmerztherapie mit Metamizol und Ibuprofen sei nicht ausreichend. Der Dienstarzt lässt der als „schwierig“ wahrgenommenen Patientin eine rote Placebo-Tablette aushändigen und bittet den Pfleger ihr zu erklären, dass diese „Spezialmedikation" schon jedem geholfen habe. In der Morgenvisite berichtet sie begeistert über die gute Wirkung und wünscht das Medikament 
nun auch tagsüber zu erhalten, sodass ihr weiterhin die Placebo-Tabletten verabreicht werden. Im Entlassgespräch fragt die Patientin dann interessiert nach dem Namen des Präparates und bittet um Erwähnung im Arztbericht um sich das Medikament für Notfälle besorgen zu können. Auch die gute Wirkung der Placebobehandlung und das lange und intensive Gespräch über die Natur der wirkstofffreien Tablette kann das gestörte Vertrauen der Patientin in das Behandlungsteam nach der Aufklärung nicht reparieren.

Dieses hypothetische Beispiel verdeutlicht gleichsam, wie wirksam Placebos sein können, aber auch das damit verbundene ethische Dilemma. Dieser Beitrag fasst aktuelle Befunde zu den psychologischen und neurobiologischen Mechanismen von Placeboeffekten und deren Einfluss auf aktive pharmakologische und andere schmerztherapeutische Behandlungen zusammen und zeigt auf, wie Placeboeffekte sogar ganz ohne die Anwendung von Scheinmedikamenten genutzt werden können, um die Wirksamkeit und Verträglichkeit von schmerztherapeutischen Behandlungen zu verbessern.

\section{Von der Wissenschaft in die Praxis}

Als Placeboeffekte bezeichnet man positive physiologische oder psychologische Veränderungen, die nach einer Einnahme von Medikamenten ohne spezifischen Wirkstoff oder Scheineingriffen auftreten. Zu diesen zählen der natürliche Verlauf einer Erkrankung, statistische Phänomene und die sogenannte Placeboantwort. Diese beschreibt Symptomveränderungen, welche durch die Erwartungshaltung von Patienten bezüglich der Wirkung einer Therapie, Lernprozesse sowie die Behandler-Patienten-Kommunikation hervorgerufen werden (zusammengefasst in [1]). Wie Untersuchungen der letzten Jahrzehnte demonstrieren (zusammengefasst in [2]), unterliegen der Placeboantwort komplexe neurobiologische und physiologische Vorgänge.

Essentiell für die klinische Nutzung von Placeboeffekten ist das Verständnis, dass Placeboantworten nicht nur im Rahmen von wissenschaftlichen Placebobehandlungen auftreten, sondern auch im Rahmen pharmakologischer oder anderer spezifischer Behandlungen den Erfolg einer Therapie substantiell beeinflussen können. Dieses Potenzial ist mittlerweile auch metaanalytisch belegt [3]. Dem gegenüber stehen sogenannte Noceboantworten, unter denen man das Auftreten oder die Verschlimmerung von Symptomen oder unerwünschten Wirkungen zusammenfasst, die durch eine negative Erwartung oder Vorerfahrung, aber nicht durch die medizinische und/oder pharmakologische Behandlung selbst entstehen. Auch hier belegen Metaanalysen, dass ein großer Anteil von Nebenwirkungen nicht auf die pharmakologische Substanz selbst, sondern auf die damit verknüpfte Vorerfahrung oder Erwartung zurückzuführen ist (zusammengefasst in $[4,5])$. In den folgenden Absätzen sollen die psychologischen und neurobiologischen Grundlagen von Placebo- und Noceboantworten erläutert und klinische Implikationen erörtert werden.

\section{Die Säulen des Placeboeffektes: Erwartungs- und Lernmechanismen}

Es ist naheliegend, dass eine wirkstofffreie Substanz oder eine Scheinintervention mit keiner direkten Wirkung zusammenhängt. Dennoch zeigen klinische und experimentelle Studien in Kontroll-
Armen häufig Placeboeffekte. Insbesondere im Bereich der Schmerztherapie sind diese oft so groß, dass für vielversprechende neue Substanzen oder Therapieverfahren der angestrebte Wirksamkeitsnachweis (gegenüber Placebo) häufig nicht gelingt. Umso wichtiger ist es, die zugrundeliegenden Mechanismen und Einflussfaktoren dieses Phänomens besser zu verstehen.

Basierend auf intensiven Forschungsaktivitäten der letzten beiden Jahrzehnte haben sich 2 eng verbundene psychologische Schlüsselmechanismen bei der Entstehung von Placeboantworten identifizieren lassen: Erwartungs- und Lernmechanismen [6].

Individuelle Behandlungserwartungen und -überzeugungen entstehen auf vielfältige Art und Weise und können auch bewusst induziert werden. Die wesentlichen Determinanten von Erwartungen sind Vorinformationen, die eine Vorhersage über den Behandlungserfolg erlauben. Empirisch gut belegte Induktoren von Behandlungserwartungen sind verbale Instruktionen (z. B. durch den Arzt/Ärztin, Apotheker, etc.), frühere Behandlungserfahrungen, sowie die soziale Beobachtung von Behandlungserfolgen/-misserfolgen bei Mitpatienten oder dem sozialen Umfeld. Die Placeboanalgesie, also die Linderung von Schmerzen durch Placeboeffekte, ist ein besonders gut untersuchtes Phänomen, auf das wir im Folgenden näher eingehen werden. Viele Studien belegen, dass experimentell induzierte Erwartungen (z. B. über verbale Information) zu einer unterschiedlichen Schmerzlinderung, erhöhten Kontrollüberzeugung und Reduktion von Angst und Stress führen können [7]. Aber auch verschiedene kontextuelle Aspekte wie der Arztkittel, die Gestaltung der Behandlungsumgebung [8] oder ganz direkte Charakteristika der Behandlung (Injektion, Tablette und dessen Form und Farbe [9]) können Placeboeffekte beeinflussen. Während in experimentellen Studien gezielt einzelne Wirkkomponenten der Erwartungsbildung moduliert werden, ist die individuelle Erwartung von Patienten im klinischen Alltag häufig multifaktoriell geprägt. Die positive Behandlungserwartung bspw. eines Patienten, der von wirkstofffreien Präparaten im Kontext der Homöoopathie profitiert, ist häufig durch die Überzeugungen von sozialen Peers, einer intensiven Behandler-Patienten-Kommunikation, früheren Lernerfahrungen, sowie den Überzeugungen des Behandlers geprägt.

Dass der Behandlungskontext - und damit auch die behandelnde Person selbst - unmittelbar Teil des Wirkungsmechanismus einer Placeboantwort sein kann, demonstrieren anschaulich sogenannte „Open-Hidden-Paradigmen“ ( $\mathbf{A b b}$. 1): Das Prinzip ist denkbar einfach. Während der Infusion eines Analgetikums z. B. in der Behandlung postoperativer Schmerzen) ist entweder ein Behandler anwesend oder ein Computer übernimmt die Steuerung der Infusion ohne Behandler-Patientenkontakt. Ähnliche Studien existieren auch zur Kontaktzeit des Behandlers während (z. B. einer Sham-Akupunktur). Die Studien zeigen, dass allein die Anwesenheit und Zuwendung, die ein Behandler dem Patienten während einer Behandlung zuteilwerden lässt, den analgetischen Effekt signifikant steigern und auch die Symptomschwere verschiedener Erkrankungen (z. B. Idiopathisches Parkinson-Syndrom) lindern kann (zusammengefasst in [10]).

Wie ausgeprägt eine solche Placeboantwort ist, hängt sowohl von der Erfahrbarkeit der Behandlung als auch vom Symptomkomplex ab: Die Effektstärken scheinen umso größer je subjektiver der Zielparameter und je erfahrbarer die Verabreichung oder Scheinintervention. So ist sie besonders groß bei subjektiv erlebten Symptomen wie Schmerz oder Depressivität. Eine wirkstofffreie Infusion be- 


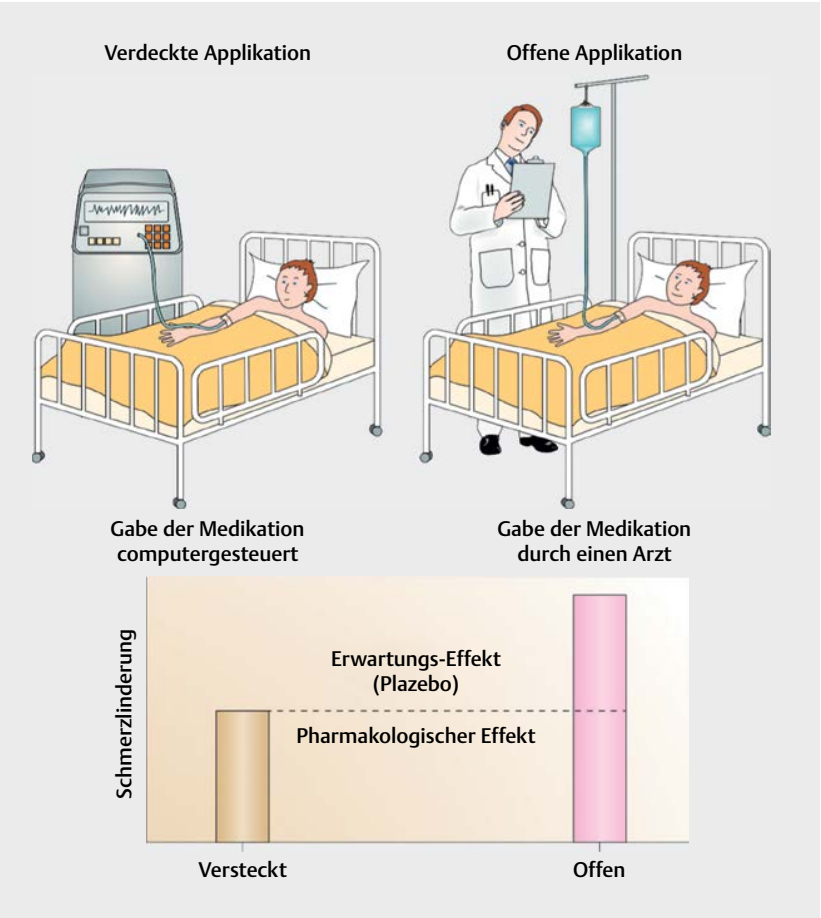

- Abb. 1 Open-Hidden-Paradigmen. Die offene Gabe eines Analgetikums als Infusion durch einen Arzt erzeugt einen Placeboeffekt durch Induktion einer positiven Erwartungshaltung an die Wirkung des Medikamentes. Somit wirkt sie stärker schmerzlindernd als die Gabe desselben Medikaments, das computergesteuert verabreicht wird. Quelle: [Enck P, Bingel U, Schedlowski M et al. The placebo response in medicine: minimize, maximize or personalize? Nat Rev Drug Discov 2013; 12: 191-204. Im Internet: http://www.nature. com/articles/nrd3923] [rerif].

wirkt bspw. eine deutlichere Schmerzlinderung als eine einfache Placebo-Tablette [11]. Dennoch finden sich auch in Organsystemen, die weniger subjektiv wahrgenommen werden, Placeboeffekte wieder. So werden bspw. Placeboantworten des Immunsystems beobachtet, welche allerdings allein durch verbal induzierte Erwartungsmechanismen nicht hinreichend erklärt werden können [12]. Vielmehr kommt ein weiterer Schlüsselmechanismus für Placeboantworten zum Tragen: Die klassische Konditionierung, oder spezifischer ausgedrückt: Die Vorerfahrung mit einem Medikament, die auch als „pharmakologische Konditionierung“ bezeichnet wird [6].

Das Konzept der pharmakologischen Konditionierung, das in - Abb. 2 dargestellt ist, besteht aus der wiederholten gleichzeitigen Präsentation eines neutralen Stimulus (z. B. die Farbe oder der Geschmack einer Tablette) mit der pharmakologischen Wirkung des Präparates (z. B. Schmerzlinderung, entspricht dem unkonditionierten Stimulus) zu einer konditionierten Reaktion, die - einmal erlernt - auch allein durch das wirkstofffreie Präparat (also dem konditionierten Stimulus) ausgelöst werden kann [6, 13, 14]. Eine solche konditionierte analgetische Reaktion auf eine Placebogabe nach wiederholter Gabe des Verum-Analgetikums wurde sowohl für Opioid- als auch für Nicht-opioid-Analgetika demonstriert $[15,16]$. Solche pharmakologisch konditionierten Reaktionen wurden insbesondere in den Bereichen gezeigt, in denen sich durch verbale Instruktion allein keine Placeboanwort auslösen ließ - wie im Immun- und endokrinen System. So gelang es bspw. sogar bei

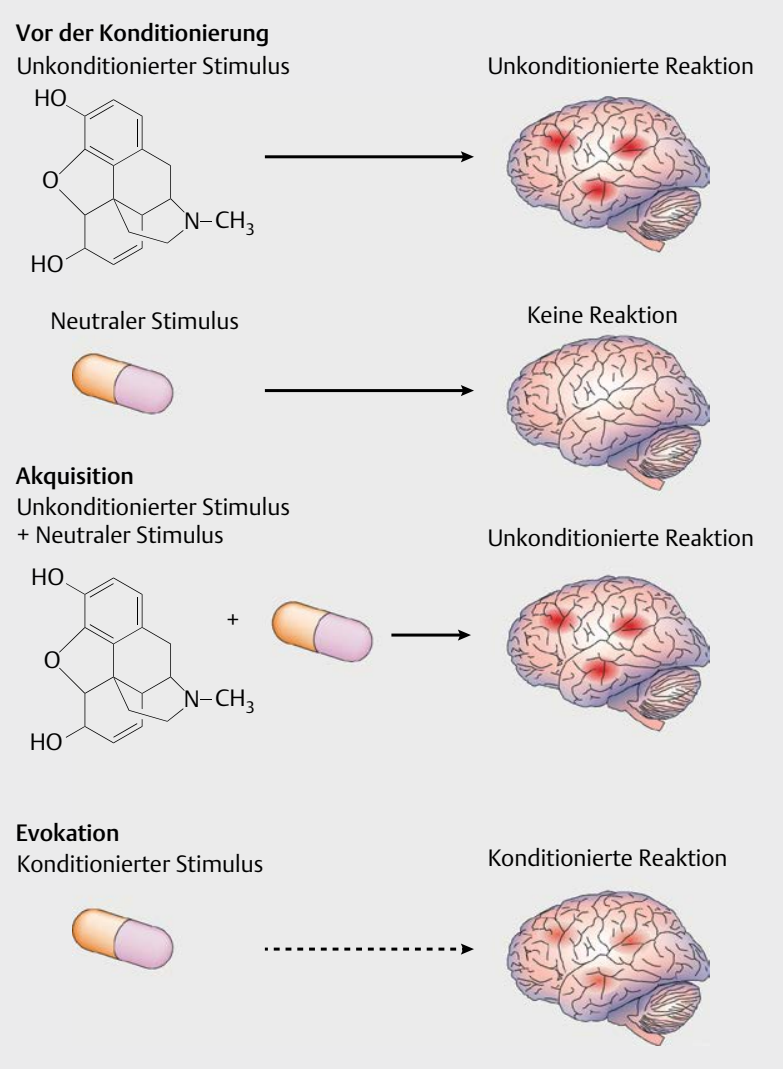

Abb. 2 Pharmakologische Konditionierung. Ein unkonditionierter Stimulus, z. B. ein Analgetikum führt zu einer nachweisbaren Wirkung (unkonditionierte Reaktion). Ein neutraler Stimulus wie eine Tablettenhülse ohne Wirkstoff führt hingegen zu keiner Wirkung. Dennoch kann die wiederholte gleichzeitige Präsentation eines neutralen Stimulus mit dem unkonditionierten Stimulus zu einer konditionierten Reaktion führen, die später auch allein durch den neutralen Stimulus (wirkstofffreies Präparat) (i. S. eines konditionierten Stimulus) ausgelöst werden kann. Quelle: [Enck P, Bingel U, Schedlowski $\mathrm{M}$ et al. The placebo response in medicine: minimize, maximize or personalize? Nat Rev Drug Discov 2013; 12: 191-204. Im Internet: http://www.nature.com/articles/nrd3923] [rerif].

nierentransplantierten Patienten durch klassische Konditionierung eine Immunsuppression mit Ciclosporin A oder Tacrolimus durch intermittierend verabreichte Placebo-Kapseln aufrecht zu erhalten und unerwünschte Arzneimittelreaktionen zu vermindern [14]. Patienten mit konditionierter Placeboantwort zeigten innerhalb der Placebo-Kapsel-Anwendung reduzierte Konzentrationen modulierender Zytokine (nachgewiesen durch IL-2-mRNA und y-IFN-mRNA) sowie eine T-Zell-Suppression. Während die Mechanismen der Placeboanalgesie von allen Placeboantworten am besten verstanden sind, gewinnen aktuelle Forschungsprojekte auch zunehmend ein Verständnis der neurobiopsychologischen Korrelate von Placeboantworten in anderen körperlichen Systemen. Hierzu werden auch peripher-physiologische Systeme zahlreicher Organstrukturen mit einbezogen. Hierbei wird deutlich, dass Placeboantworten als ein fast ubiquitär vorkommendes Phänomen beschrieben werden können [1], wie $>$ Abb. 3 veranschaulicht.

Übertragen auf das einleitende Fallbeispiel lassen sich auch hier Faktoren der nun bekannten Mechanismen identifizieren: Grund- 


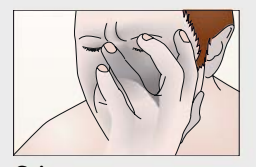

Stimmung

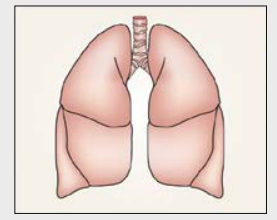

Atmung

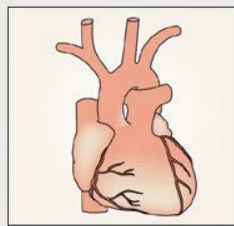

Kardiovaskuläres System
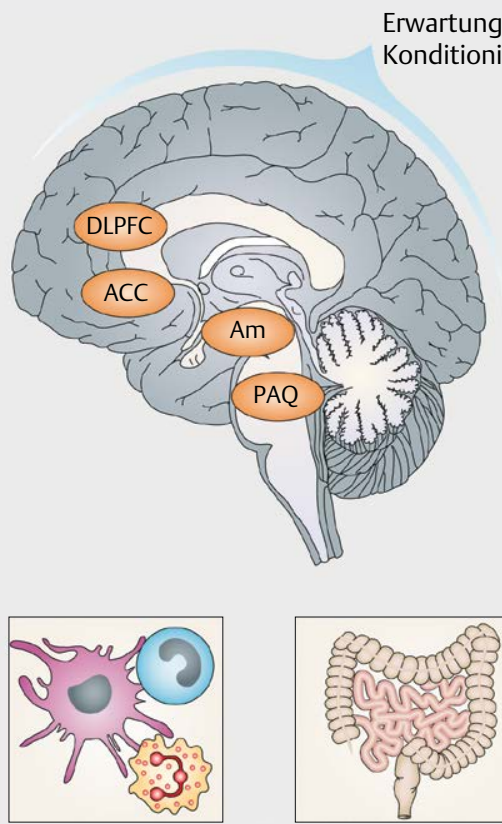

Immunsystem

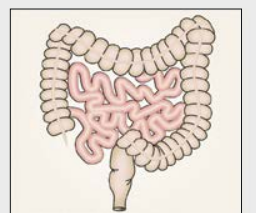

Gastrointestinale System

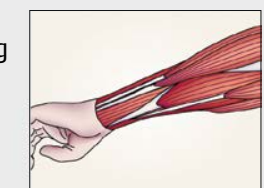

Motorisches System

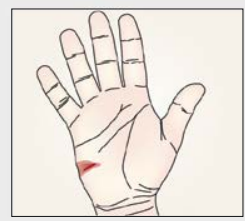

Schmerz

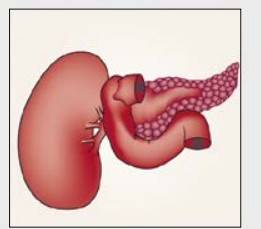

Endokrines System

Abb. 3 Placeboantworten als psychoneurobiologisches Phänomen. Placeboantworten betreffen viele körpereigene Systeme. Quelle [Enck P, Bingel U, Schedlowski M et al. The placebo response in medicine: minimize, maximize or personalize? Nat Rev Drug Discov 2013; 12: 191-204. Im Internet: http://www.nature.com/articles/nrd3923] [rerif].

lage einer möglichen Placeboantwort ist z. B. die Tatsache, dass es sich um eine rote Tablette handelt. Die Einnahme einer roten Tablette allein führt wohlmöglich durch eine bereits bestehende Konditionierung der Patientin zu einer schmerzlindernden Wirkung. Die verbale Instruktion, dass es sich dabei um ein „Spezialmedikament" handele, das mit großem Erfolg selbst bei medizinisch herausfordernden Fällen eingesetzt werde, kann eine entsprechende positive Erwartungshaltung erzeugt haben. Zu guter Letzt legen die sog. Open-Hidden-Paradigmen nahe, dass die Anwesenheit und die Bereitschaft des Pflegenden, die Placebotablette zu erklären, eine mögliche Wirkung induzieren kann. All diese Faktoren können über die Mechanismen der Erwartung und Konditionierung eine Placeboanalgesie auslösen.

\section{Neurobiologische Korrelate der Placeboanalgesie}

Aktuellen Modellen der Placeboanalgesie zufolge, beruht diese auf der kognitiv getriggerten (d. h. Entwicklung einer Erwartungshaltung) Aktivierung des deszendierenden, schmerzhemmenden Systems, wobei dem dorsolateralen prefrontalen Kortex (DLPFC), dem rostralen anterioren Cingulum ( $\mathrm{rACC}$ ) und subkortikalen Kerngebieten wie dem periaquäduktalen Grau (PAG) eine Schlüsselrolle zukommen [17]. Die Aktivierung dieses schmerzhemmenden Systems bedingt eine verminderte Aktivierung schmerzrelevanter Areale, wie dem insulären, und somatosensorischen Kortex. Untersuchungen mittels spinaler funktioneller MRT (fMRT) legen nahe, dass dies auf eine Modulation der nozizeptiven Signalverarbeitung bereits auf Höhe des spinalen Hinterhorns zurückzuführen ist [18]. Sowohl phar- makologische Interventionen mit dem Opioidantagonisten Naloxon in Kombination mit fMRT, als auch Untersuchungen mit Opioid-Liganden-Positronen-Emissions-Tomographie (PET, z. B. [11C]Carfentanil-PET) belegen die substantielle Beteiligung des endogenen Opioidsystems für die Schmerzhemmung während der Placeboanalgesie [19]. Aktuellste Befunde deuten darauf hin, dass unter bestimmten Umständen (wiederholte Vorbehandlung mit einem Nicht-Opioid-Analgetikum) auch das körpereigene Cannabinoidsystem an der Placeboanalgesie beteiligt sein kann [15].

\section{Individuelle Unterschiede der Placeboantwort}

Eine große Herausforderung bei der gezielten klinischen Anwendung von Placeboeffekten ist ihre große interindividuelle Varianz. Diese reicht bspw. bei der Placeboanalgesie von einer geringen Schmerzlinderung bis zur kompletten Schmerzfreiheit selbst stark chronifizierter Schmerzsyndrome. Diese Varianz spiegelt sich auch in den Placeboarmen der klinischen Studien wider. Ein Schwerpunkt aktueller Forschungsbemühungen ist daher die Charakterisierung von psychologischen, physiologischen, genetischen und anderen Prädiktoren der individuellen Placeboantwort. Bislang ist das Wissen bzgl. potentieller Prädiktorvariablen für eine Placeboantwort noch lückenhaft. So scheinen psychologische Variablen wie die Ängstlichkeit von Probanden oder Patienten, das Ausmaß an Depressivität oder Optimismus einen Teil der Varianz in der Placeboantwort zu erklären. Auch die individuelle genetische Ausstattung [20] und die inviduelle Hirnanatomie beeinflussen die individuelle Placeboantwort [21]. Vor dem Hintergrund, dass Placeboeffekte substantiell zum therapeutischen Outcome vieler Behandlungen 
beitragen, ist der weitere Erkenntnisgewinn bzgl. möglicher Prädiktoren von Placeboantworten von großer Bedeutung, sowohl für den klinischen Alltag, als auch für eine mögliche Stratifizierung in klinischen Studien.

\section{Die klinische Anwendbarkeit von Placeboeffekten}

Durch positive Erwartungen begünstigte Placeboantworten können auch ganz ohne die Gabe eines Placebos auftreten und die medizinische Behandlung beeinflussen oder über die rein pharmakologische Wirkung hinaus verbessern. Im Kontrast dazu können allerdings auch negative Erwartungen Medikamenteneffekte mildern oder aufheben. Dies zeigte eine Studie, in der das potente Opioidanalgetikum Remifentanil in 3 Erwartungsbedingungen eingesetzt wurde: Ohne Erwartung als verdeckte Infusion, mit verbundener positiver Erwartung und mit negativer Erwartung [7]. Alle Erwartungsinduktionen geschahen dabei über eine verbale Instruktion in Kombination mit einer Konditionierungsphase. In dieser Studie verdoppelte sich der analgetische Effekt allein durch das Wissen der Versuchsteilnehmer, dass sie das Analgetikum während eines experimentellen Schmerzreizes erhielten. Versuchsteilnehmer, denen allerdings eine negative Instruktion kommuniziert wurde (keine Therapie, aber schmerzhafter Reiz), zeigten keinen lindernden Effekt der pharmakologisch wirksamen Substanz, obwohl die identische Dosis des Medikamentes verabreicht wurde. Parallele fMRT-Untersuchungen belegten die Erwartungsmodulation der Analgesie in klassischen Schmerzarealen. Diese an gesunden Versuchsteilnehmern durchgeführte Studie hat deutliche klinische Implikationen: Negative Erwartungen, komorbide Angst und Depression sind insbesondere bei chronischen Schmerzpatienten häufig und sollten im therapeutischen Kontext berücksichtigt werden. Es liegt nahe, dass also pharmakologische Schmerztherapien mit negativen Vorerfahrungen und Gedanken interferieren und somit schlechter wirken.

Auch in der Behandlung episodischer Schmerzerkrankungen, wie der Migräne, sind vergleichbare Befunde zu finden: In einer prospektiven, randomisierten, placebo-kontrollierten Untersuchung wurden Patienten mit episodischer Migräne rekrutiert und 6 konsekutive Migräneattacken mit einer Studienmedikation behandelt [22]. Diese bestand entweder aus Rizatriptan $10 \mathrm{mg}$ oder Placebo, die jeweils entweder als „Placebo“, „Placebo oder „Rizatriptan“ oder „Rizatriptan“ beschriftet war, sodass der Einfluss unterschiedlicher Erwartungen von negativ bis positiv auf die Wirksamkeit der Studienmedikation untersucht werden konnte. Es zeigte sich eine leicht größere Wirkung von Rizatriptan gegenüber Placebo. Allerdings ergab sich sowohl für Placebo als auch für Rizatriptan eine statistisch signifikante Modulation durch die Beschriftung - mit zunehmender Wirksamkeit bei zunehmender positiver Erwartung. Hierbei war die Wirkung von Rizatriptan, welches als Placebo dargeboten wurde, vergleichbar mit Placebo, welches als Rizatriptan dargeboten wurde. Bemerkenswerterweise war auch die Gabe von Placebos, welche wahrheitsgemäß als Placebos beschriftet wurden, wirksamer als eine Nichtbehandlung, was möglicherweise auf konditionierte Effekte zurückzuführen ist, da die Patienten nicht naiv für die Behandlung mit Triptanen oder anderen Analgetika waren.

Diese Studien verdeutlichen exemplarisch, dass Placebo- und Noceboeffekte, bzw. die Effekte positiver und negativer Erwartung und
Behandlungsvorerfahrung die Wirksamkeit von aktiven pharmakologischen (und anderen) schmerzmedizinischen Behandlungen stark beeinflussen können. Wie Untersuchungen zeigen, können insbesondere Vorerfahrungen dabei eine bedeutende Rolle spielen und über Placebo- und Noceboeffekte die Wirkung auch neuartiger Therapien beeinflussen [23]. Sie geben Anlass zur Diskussion, wie Placeboantworten im klinischen Umfeld nutzbringend eingesetzt werden können, um die Wirksamkeit und Verträglichkeit schmerztherapeutischer Behandlungen zu optimieren. Ein Ansatz, der mittlerweile auch Einzug in aktuelle Behandlungsleitlinien gefunden hat [24].

\section{Ethische und juristische Überlegungen}

Auch wenn Placebobehandlungen sowohl in experimentellen als auch in klinischen Studien zu einer Symptombesserung führen können, ist die Darreichung von reinen Placebos im praktischen Alltag aus juristischen und ethischen Gründen höchst problematisch, wie das einleitende Fallbeispiel zeigt. Das illustrierte Dilemma stand bislang einer einfachen Translation des Placeboeffektes in den klinischen Alltag im Weg: Bei der „traditionellen“ Anwendung von Placebobehandlungen galt es als notwendig, den Patienten über das Wesen der Placebobehandlung im Unklaren zu lassen. Eine vorsätzliche Täuschung ist nicht nur ethisch und juristisch fragwürdig, sondern gefährdet die Behandler-Patienten-Beziehung deutlich. Wie können diese signifikanten und klinisch relevanten Placeboeffekte dennoch in der Schmerztherapie zur Anwendung kommen? Hierzu führen wir 2 Möglichkeiten auf: (a) die Verstärkung analgetischer Effekte leitliniengerechter Schmerztherapien durch Nutzung der zugrundeliegenden Mechanismen einer Placeboantwort und (b) - bislang noch Teil investigativer Forschung - die Anwendung sogenannter Open-label Placebos.

Die Verstärkung der Wirkung offen verabreichter Analgetika beruht auf den Befunden der zuvor vorgestellten Open-Hidden-Paradigmen (siehe oben) und der Bedeutung der Behandlungserwartung für den analgetischen Effekt einer Pharmakotherapie. Ziel ist es, dass Patienten ihre Medikamente so bewusst wie möglich einnehmen. Je stärker die Verabreichung eines Arzneimittels wahrgenommen wird (z. B. Sehen, Riechen, Schmecken, Berühren und Information), desto besser kann der Placeboeffekt genutzt werden. Weiterhin kann es förderlich sein, Patienten intensiver und gezielter über ihre Erkrankung und Behandlungen aufzuklären, um positive Erwartungen zu wecken und negative Erwartungen und Befürchtungen zu vermeiden. Die häufig geteilte Ansicht, verdeckte Behandlungen kämen nur im Rahmen experimenteller Untersuchungen vor, ist nicht haltbar. Es ist davon auszugehen, dass Patienten oft nur ein eingeschränktes Wissen bezüglich einer Pharmakotherapie, deren Wirkweise und der zu erwartenden Wirkung haben [25]. Daher kann bereits durch eine empathische und fokussierte Aufklärung möglicherweise eine Placeboantwort induziert werden. Nicht zuletzt, könnten auch Strategien des sozialen Lernens z. B. i.R. eines sog. Peer-toPeer Coachings genutzt werden. Weitere praktische Ansätze, die sich aus der bestehenden Literatur ergeben fasst $>$ Tab. 1 zusammen. Auch wenn diese Maßnahmen bis dato nicht in großen kontrollierten klinischen Studien untersucht wurden, legen experimentelle und kleinere klinische Studien deren Nutzen für den Patienten nahe. 
- Tab. 1 Praktische Maßnahmen zur klinischen Nutzung von Placeboeffekten in der Schmerzmedizin.

\begin{tabular}{|c|c|c|}
\hline & Erwartungsmechanismen & Konditionierungsprozesse \\
\hline Allgemeines: & \multicolumn{2}{|c|}{$\begin{array}{l}\text { - Komorbiditäten berücksichtigen (Depression, Angststörung) und behandeln, da diese Placebo-Effekte beeinträchtigen können } \\
\text { - Empathische und authentische Arzt-Patienten-Interaktion } \\
\text { - Behandlungskontext berücksichtigen und optimieren (Positionierung, Wartezimmer) }\end{array}$} \\
\hline Fördern Sie: & $\begin{array}{l}\text { - Verständliche Aufklärung z. B. zu Pharmakothera- } \\
\text { pien unter Einbezug der } \\
\text { - Grunderkrankung } \\
\text { - Komorbiditäten (insb. Angst) } \\
\text { - individuellen Patientensituation } \\
\text { - mögliche Vorerfahrungen } \\
\text { - Realistische Information über } \\
\text { - individuelle Therapieziele } \\
\text { - Wirkungsmechanismen } \\
\text { - erwartende Wirkung } \\
\text { - Nebenwirkungen }\end{array}$ & $\begin{array}{l}\text { - Kopplung der pharmakologischen Therapie an positive sensorische } \\
\text { Stimuli wie Geschmack, Geruch, Gefühl (Berücksichtigung der } \\
\text { Vorinformation des Patienten) } \\
\text { - Verbindung pharmakologischer Therapien mit nicht-pharmakologi- } \\
\text { schen, positiv assoziierten Maßnahmen (z. B. Entspannungstechnik) } \\
\text { - Individueller Heilversuch: Intermittierende Placebo-Gabe zur Dosisre- } \\
\text { duktion bei Langzeittherapie }\end{array}$ \\
\hline Vermeiden Sie: & $\begin{array}{l}\text { - unrealistische Aufklärung (Bestärkung von Angst } \\
\text { und Unsicherheit) } \\
\text { - verdeckte Medikamentengaben }\end{array}$ & $\begin{array}{l}\text { - Beginn oder Fortführung einer negativ erlernten oder assoziierten } \\
\text { Therapie (negative Vorerfahrung) }\end{array}$ \\
\hline
\end{tabular}

\section{Open-Label Placebos}

Eine neuartige, wenngleich aktuell mehr akademische als klinische Nutzung von Placeboeffekten eröffnen offene Placeboanwendungen. Randomisierte, kontrollierte Studien der letzten 5 Jahre legen nahe, dass eine Verschleierung der Wirkstofffreiheit eines Placebos für die Ausprägung einer Placeboantwort gar nicht notwendig sein könnte. Interessanterweise scheint eine offen kommunizierte Gabe von wirkstofffreien Tabletten oder Kapseln, sogenannten open-label Placebos (OLP), ebenfalls zu positiven Veränderungen hinsichtlich der untersuchten Beschwerdebilder zu führen [26]. Die bis heute verfügbaren Studien beruhen allerdings überwiegend auf kleineren Stichproben zwischen 20 und 120 Patienten. Sie stellen aber insgesamt einen beeindruckenden ersten Wirknachweis für den klinischen Nutzen von Open-label Placebos dar. Die Ergebnisse weisen auf eine signifikante Reduktion der Symptomlast bei Schmerzerkrankungen (episodische Migräne [22] chronischer Rückenschmerz [27, 28], Reizdarmsyndrom [29]), neuropsychiatrischen Syndromen (chronisches Fatigue-Syndrom bei Tumorerkrankungen [30], Aufmerksamkeitsdefizit-Hyperaktivitäts-Syndrom [31], Depression [32]) und bei allergischer Rhinitis [33] hin. Die beobachteten Effektgrößen sind dabei vergleichbar mit denen, die im Rahmen traditioneller Placebo-Anwendungen belegt sind. Diese Studien legen nahe, dass auch bei OLP subjektive und patientenberichtete Outcomes (wie bspw. das Schmerzempfinden) stärker beeinflusst werden als objektive Outcomes (wie Bewegungsparameter der Wirbelsäule, Laborparameter). Große Studien müssen jedoch die Replizierbarkeit der Effekte in kontrollierten Settings zunächst prüfen und die grundlegenden Wirkfaktoren systematisch untersuchen, da das Verständnis der zugrundeliegenden Mechanismen derzeit noch unvollständig ist. Vielversprechende Anwendungsbereiche sind stark schwankende und individuell belastende Beschwerden wie gastrointestinale, neurologische, psychosomatische und Schmerzsymptome. Ebenso erscheint die Reduktion von Nebenwirkungen oder Absetzerscheinungen bei Pharmakotherapien sinnvoll. Ob OLPs in die klinische Routine übertragbar sind und wie Patienten dabei optimal aufgeklärt und an einer Behandlungsentscheidung beteiligt werden können, bleibt zu prüfen.

\section{FAZIT}

Unter einer Placeboantwort versteht man positive psychologische oder physiologische Veränderungen, die durch Erwartungs- und Konditionierungsprozesse induziert werden und mit messbaren neurobiologischen und peripher-physiologischen Vorgängen einhergehen. Auch wenn Placeboantworten traditionell im Zusammenhang mit der Einnahme von Scheinmedikamenten untersucht wurden, treten diese auch ganz ohne die Verabreichung von Placebos auf und beeinflussen die Wirksamkeit und Verträglichkeit jeder medizinischen Behandlung. Die Darreichung von reinen Placebos ist im praktischen Alltag sowohl aus juristischen und ethischen Gründen höchst problematisch, jedenfalls dann, wenn der Patient irregeführt wird und nicht über das Wesen der Placebobehandlung informiert wird. Die direkte klinische Nutzung von Placeboeffekten im Rahmen einer offenen PlaceboAnwendung (sogenannte Open-label Placebos), bei der Patienten über die Wirkstofffreiheit der Placebos informiert sind, ist derzeit Bestandteil zahlreicher wissenschaftlicher Untersuchungen und könnte zukünftig Potenzial als Add-onTherapie oder im Rahmen von Dosisreduktionen bieten [34]. Zum jetzigen Zeitpunkt besteht die unbedingte Empfehlung, die zugrundeliegenden Mechanismen von Placeboantworten gezielt im klinischen Alltag zu nutzen, um die Wirksamkeit, Verträglichkeit und Compliance bestehender pharmakologischer und anderer Therapien zum Wohle des Patienten zu optimieren. Dazu zählen insbesondere die gezielte Modulation von Erwartungs- und Lernprozessen im Rahmen der Behandlung, die sich durch eine patientenzentrierte Information und einen authentisch-empathischen Kommunikationsstil erreichen lässt. 


\section{Interessenkonflikt}

Die Autorinnen/Autoren geben an, dass kein Interessenkonflikt besteht.

\section{Literatur}

[1] Enck P, Bingel U, Schedlowski $\mathrm{M}$ et al. The placebo response in medicine: minimize, maximize or personalize? Nat Rev Drug Discov 2013; 12 : 191-204. Im Internet: http://www.nature.com/articles/nrd3923

[2] Schedlowski M, Enck P, Rief W et al. Neuro-bio-behavioral mechanisms of placebo and nocebo responses: Implications for clinical trials and clinical practice. Pharmacol Rev 2015; 67: 697-730. Im Internet: https://www.ncbi.nlm.nih.gov/pubmed/26126649

[3] Vase L, Petersen GL, Riley JL, et al. Factors contributing to large analgesic effects in placebo mechanism studies conducted between 2002 and 2007. Pain 2009

[4] Bingel U. Avoiding nocebo effects to optimize treatment outcome. JAMA - J Am Med Assoc 2014; 312: 693-694

[5] Mitsikostas DD. Nocebo in headaches: Implications for clinical practice and trial design. Curr Neurol Neurosci Rep 2012; 12: 132-137

[6] Doering BK, Rief W. Utilizing placebo mechanisms for dose reduction in pharmacotherapy. Trends Pharmacol Sci 2012; 33: 165-172

[7] Bingel U, Wanigasekera V, Wiech $\mathrm{K}$ et al. The Effect of Treatment Expectation on Drug Efficacy: Imaging the Analgesic Benefit of the Opioid Remifentanil. Sci Transl Med 2011; 3: 70. Im Internet: http:// www.ncbi.nlm.nih.gov/pubmed/21325618

[8] Ulrich RS. View through a window may influence recovery from surgery. Science (80- ) 1984; 224: 420-421

[9] Meissner K, Linde K. Are Blue Pills Better Than Green? How Treatment Features Modulate Placebo Effects.In: International Review of Neurobiology. Academic Press Inc; 2018: 357-378

[10] Colloca L, Lopiano L, Lanotte M et al. Overt vs. covert treatment for pain, anxiety, and Parkinson's disease. Lancet Neurol 2004; 3: 679-684. Im Internet: https://linkinghub.elsevier.com/retrieve/pii/ S1474442204009081

[11] Vase L, Riley JL, Price DD. A comparison of placebo effects in clinical analgesic trials vs. studies of placebo analgesia. Pain 2002; 99: 443-452

[12] Stewart-Williams S, Podd J. The Placebo Effect: Dissolving the Expectancy vs. Conditioning Debate. Psychol Bull 2004; 130: 324-340. Im Internet: http://doi.apa.org/getdoi.cfm?doi = 10.1037/00332909.130.2.324

[13] Jensen K, Kirsch I, Odmalm S et al. Classical conditioning of analgesic and hyperalgesic pain responses without conscious awareness. Proc Natl Acad Sci U S A 2015; 112: 7863-7867. Im Internet: http://www. ncbi.nlm.nih.gov/pubmed/25979940

[14] Kirchhof ], Petrakova L, Brinkhoff A et al. Learned immunosuppressive placebo responses in renal transplant patients. Proc Natl Acad Sci USA 2018; 115: 4223-4227

[15] Benedetti F, Amanzio M, Rosato $R$ et al. Nonopioid placebo analgesia is mediated by CB1 cannabinoid receptors. Nat Med 2011; 17: 1228-1230

[16] Amanzio M, Benedetti F. Neuropharmacological dissection of placebo analgesia: Expectation- activated opioid systems vs. conditioningactivated specific subsystems. J Neurosci 1999; 19: 484-494

[17] Wager TD, Atlas LY. The neuroscience of placebo effects: Connecting context, learning and health. Nat Rev Neurosci 2015; 16: 403-418. Im Internet: https://www.ncbi.nlm.nih.gov/pubmed/26087681
[18] Eippert F, Finsterbusch J, Bingel U et al. Direct evidence for spinal cord involvement in Placebo Analgesia. Science (80- ) 2009; 326: 404

[19] Scott D], Stohler CS, Egnatuk CM et al. Placebo and nocebo effects are defined by opposite opioid and dopaminergic responses. Arch Gen Psychiatry 2008; 65: 220-231

[20] Kaptchuk T], Miller FG. Placebo effects in medicine. N Engl ] Med 2015; 373: 8-9. Im Internet: http://www.nejm.org/doi/10.1056/ NEJMp1504023

[21] Wager TD, Atlas LY, Leotti LA et al. Predicting individual differences in placebo analgesia: Contributions of brain activity during anticipation and pain experience. J Neurosci 2011; 31: 439-452

[22] Kam-Hansen S, Jakubowski M, Kelley JM et al. Altered placebo and drug labeling changes the outcome of episodic migraine attacks. Sci TransI Med 2014; 6: 218. Im Internet: http://stm.sciencemag.org/cgi/ doi/10.1126/scitranslmed.3006175

[23] Zunhammer M, Ploner M, Engelbrecht $C$ et al. The effects of treatment failure generalize across different routes of drug administration. Sci Transl Med 2017; 9: 1-8

[24] Laubenthal H, Neugebauer E. S3-Leitlinie „Behandlung akuter perioperativer und posttraumatischer Schmerzen“. 2009. Im Internet: http://www.awmf.org/uploads/tx_szleitlinien/001-025I_S3_Behandlung_akuter_perioperativer_und_posttraumatischer_Schmerzen_abgelaufen.pdf

[25] Bodenheimer T. Transforming Practice. N Engl J Med 2008; 359: 2086-2089. Im Internet: http://www.nejm.org/doi/abs/10.1056/ NEJMp0805631

[26] Charlesworth JEG, Petkovic G, Kelley JM et al. Effects of placebos without deception compared with no treatment: A systematic review and meta-analysis. J Evid Based Med 2017; 10: 97-107. Im Internet: http://doi.wiley.com/10.1111/jebm.12251

[27] Kleine-Borgmann J, Schmidt K, Hellmann A et al. Effects of open-label placebo on pain, functional disability, and spine mobility in patients with chronic back pain: A randomized controlled trial. Pain 2019; 160: 2891-2897

[28] Carvalho C, Caetano JM, Cunha L et al. Open-label placebo treatment in chronic low back pain: A randomized controlled trial. Pain 2016; 157: 2766-2772. Im Internet: http://dx.doi.org/10.1097/j. pain. 0000000000000700

[29] Kaptchuk T], Friedlander E, Kelley JM, et al. Placebos without deception: A randomized controlledtrial in irritable bowel syndrome. PLoS One 2010

[30] Zhou ES, Hall KT, Michaud AL et al. Open-label placebo reduces fatigue in cancer survivors: a randomized trial. Support Care Cancer 2019; 27: 2179-2187. Im Internet: https://www.ncbi.nlm.nih.gov/pubmed/30298411

[31] Sandler AD, Bodfish JW. Open-label use of placebos in the treatment of ADHD: a pilot study. Child Care Health Dev 2007; 34: 104-110. Im Internet: http://doi.wiley.com/10.1111/j.1365-2214.2007.00797.x

[32] Kelley JM, Kaptchuk T], Cusin C et al. Open-label placebo for major depressive disorder: A pilot randomized controlled trial. Psychother Psychosom 2012; 81: 312-314. Im Internet: http://www.ncbi.nlm.nih. gov/pubmed/22854752

[33] Schaefer M, Harke R, Denke C. Open-label placebos improve symptoms in allergic rhinitis: A randomized controlled trial. Psychother Psychosom 2016; 85: 373-374

[34] Belcher AM, Cole TO, Greenblatt AD et al. Open-label dose-extending placebos for opioid use disorder: A protocol for a randomised controlled clinical trial with methadone treatment. BMJ Open 2019; 9 